\title{
Investigating molecular alterations to profile short- and long-term recurrence-free survival in patients with primary glioblastoma
}

\author{
SARA FRANCESCHI ${ }^{1,2}$, CHIARA M. MAZZANTI ${ }^{2}$, FRANCESCA LESSI $^{2}$, PAOLO ARETINI $^{2}$, \\ FRANCESCO G. CARBONE ${ }^{1}$, MARCO LA FERLA ${ }^{2}$, CRISTIAN SCATENA ${ }^{1}$, VALERIO ORTENZI ${ }^{1}$, \\ RICCARDO VANNOZZI $^{3}$, GIOVANNI FANELLI ${ }^{3}$, FRANCESCO PASQUALETTI ${ }^{4}$, GENEROSO BEVILACQUA ${ }^{1}$, \\ KATIA ZAVAGLIA $^{1}$ and ANTONIO G. NACCARATO ${ }^{1}$

\begin{abstract}
${ }^{1}$ Department of Translational Research and of New Surgical and Medical Technologies, University Hospital of Pisa, Pisa I-56126; ${ }^{2}$ Genomic Section, Pisa Science Foundation, Pisa I-56121; ${ }^{3}$ Department of Neurosurgery, University Hospital of Pisa, Pisa I-56124; ${ }^{4}$ Radiotherapy Unit, University Hospital of Pisa, Pisa I-56126, Italy
\end{abstract}

Received January 12, 2015; Accepted August 17, 2015

DOI: $10.3892 / \mathrm{ol} .2015 .3738$

\begin{abstract}
Glioblastoma (GB) is the most aggressive type of primary brain tumor. Despite the progress in recent years regarding the diagnosis and treatment of GB, the recurrence rate remains high, due to the infiltrative and dispersive nature of the tumor, which typically results in poor patient prognosis. In the present study, 19 formalin-fixed, paraffin-embedded GB samples were selected from patients with GB tumors. The samples were classified into a short or long recurrence-free survival (RFS) group, based on the time of first recurrence of the disease in the patients. The 19 samples were molecularly characterized for mutations in the isocitrate dehydrogenase 1 (IDH1) gene, amplification of the epidermal growth factor receptor (EGFR) gene, presence of the EGFR variant III, and methylation of the promoter region of the $\mathrm{O}^{6}$-methylguanine-DNA methyltransferase (MGMT) gene. Then, the expression of 84 genes involved in cell-cell and cell-matrix interactions, and that of 84 microRNAs (miRNAs) associated with brain cancer, was profiled. In addition, a copy number variation analysis of 23 genes reported to undergo frequent genomic alterations in human glioma was also performed. Differences in the expression levels of a number of genes were detected across the short and long RFS groups. Among these genes, 5 in particular were selected, and a 5-genes combination approach was developed, which was able to differentiate between patients with short and long RFS outcome. The high levels of sensitivity and precision displayed by this 5 -genes combination approach, which were confirmed with a cross-validation method, provide a strong foundation for further validation of the involvement of the aforementioned genes in GB in a larger patient population. In
\end{abstract}

Correspondence to: Dr Sara Franceschi, Genomic Section, Pisa Science Foundation, Via Panfilo Castaldi 2, Pisa I-56121, Italy

E-mail: sara.franceschi@fondazionepisascienza.org

Key words: glioblastoma, recurrence, molecular alterations, outcome prediction, gene expression conclusion, the present study has demonstrated how the expression pattern of miRNAs and mRNAs in patients with GB defines a particular molecular hallmark that may increase or reduce the aggressive behavior of GB tumors, thus influencing the survival rates of patients with GB, their response to therapy and their tendency to suffer a relapse.

\section{Introduction}

Glioblastoma (GB) is the most common and aggressive type of malignant primary brain tumor. The current standard of care for GB, as established by Stupp et al (1), is maximal safe surgical resection, followed by temozolomide chemotherapy and radiation. This current standard therapeutic regimen has increased the median survival rate of patients with GB to 12.1-14.6 months, with a median 2-years survival rate of $26 \%$ (2). However, mortality from GB inevitably occurs, due to the recurrence or progression of the disease (3). Previous studies have indicated that recurrence of GB is unavoidable, following a median survival time of 8-9 months (3). Once a patient with GB relapses following initial surgery, radiotherapy and chemotherapy, the salvage strategies available are often limited, and the survival time is short $(4,5)$. This is most likely due to molecular and genetic alterations within the tumor itself, or as a consequence of preceding therapies (3).

Therefore, novel therapeutic approaches to treat GB, and scientific and clinical advances in the treatment of this disease are required. The identification of molecular biomarkers for the management and monitoring of patients with cancer may improve their clinical outcome. Numerous biomarker candidates have been generated by high-throughput technologies, which are powerful and promising methods for evaluating the expression of a large number of genes and detecting alterations in genome-wide expression analyses (6). An important aspect of GB invasion is the elaboration by the tumor cells of a migration-enhancing extracellular matrix (ECM), and the secretion of proteolytic enzymes that permit cell invasion through this matrix (7). This hypothesis is consistent with previous gene expression profiling studies, which identified a subset of tumors with increased expression of ECM components and 
Table I. Demographic characteristics of the patient population at the time of diagnosis, and molecular characterization of the glioblastoma tumors.

\begin{tabular}{|c|c|c|c|}
\hline Patient features at diagnosis & Overall, n (\%) & Short RFS, n (\%) & Long RFS, n (\%) \\
\hline Total (n) & 19 & 10 & 9 \\
\hline \multicolumn{4}{|l|}{ Gender } \\
\hline Female & $10(53)$ & $6(60)$ & $4(44)$ \\
\hline Male & $9(47)$ & $4(40)$ & $5(56)$ \\
\hline \multicolumn{4}{|l|}{ Age } \\
\hline Mean \pm standard deviation & $60 \pm 6.9$ & $63 \pm 6.2$ & $58 \pm 6.8$ \\
\hline$<60$ years & $9(47)$ & $4(40)$ & $5(56)$ \\
\hline$\geq 60$ years & $10(53)$ & $6(60)$ & $4(44)$ \\
\hline \multicolumn{4}{|l|}{ Molecular alterations } \\
\hline \multicolumn{4}{|l|}{ EGFR } \\
\hline wt & $10(53)$ & $7(70)$ & $3(33)$ \\
\hline ampl & $9(44)$ & $3(30)$ & $6(67)$ \\
\hline \multicolumn{4}{|l|}{ EGFRvIII in EGFR ampl } \\
\hline Yes & $3(33)$ & $1(33)$ & $2(33)$ \\
\hline No & $6(67)$ & $2(67)$ & $4(67)$ \\
\hline \multicolumn{4}{|l|}{ MGMT } \\
\hline wt & $11(58)$ & $5(50)$ & $6(67)$ \\
\hline met & $8(42)$ & $5(50)$ & $3(33)$ \\
\hline \multicolumn{4}{|l|}{ IDH1 } \\
\hline wt & $19(100)$ & $10(100)$ & $9(100)$ \\
\hline mut & $0(0)$ & $0(0)$ & $0(0)$ \\
\hline
\end{tabular}

Patients displaying EGFR wt were characterized by no alterations in the copy number of the EGFR gene, whereas those exhibiting EGFR ampl presented a high copy number of EGFR. Patients with EGFRvIII carried the constitutively active genomic deletion variant III of EGFR, characterized by the deletion of exons 2-7 in the EGFR gene. MGMT wt indicated absence of methylation in the promoter CpG islands of the MGMT gene, contrarily to the case of patients with MGMT met. Patients were classified as IDH1 wt or IDH1 mut, based on the absence or presence of mutations in the codon 132 of the IDH1 gene, respectively. RFS, recurrence-free survival; EGFR, epidermal growth factor receptor; wt, wild-type; ampl, amplification; EGFRvIII, EGFR variant III; MGMT, O(6)-methylguanine-DNA methyltransferase; met, methylation; $\mathrm{CpG}$, cytosine-phosphate-guanine; IDH1, isocitrate dehydrogenase 1; mut, mutation.

intracellular proteins associated with cell motility $(8,9)$. The interplay between the various matrix and growth factor receptors, and the activation of signaling pathways that facilitate the invasion of tumor cells, has been recently recognized as a composite, dynamic consequence of altered cell-cell adhesion, proteolytic remodeling and synthesis of ECM, and selective expression and activation of integrins (10). Furthermore, in the past few years, several signaling pathways have been associated with reduced sensitivity of GB cells to radiation and chemotherapy (11). Numerous genes involved in these pathways have been demonstrated to be regulated by microRNAs (miRNAs), which are important regulators in cancer cell biology, and promising biomarkers or therapeutic targets in GB $(11,12)$.

However, despite all the molecular information on GB gathered thus far, the optimal management of patients with GB remains elusive, due to the absence of validated data from clinical studies, and the great heterogeneity of this fragile subpopulation, in terms of their physical condition, co-morbidity status, tolerance to treatment and clinical prognosis (13). The invasive nature of GB growth and its fast proliferation rate are the major reasons for therapeutic failure in patients with GB (14). Therefore, a detailed characterization of the molecular mechanisms involved in the development of GB is required, in order to improve the accuracy of the prediction models, regarding the prognosis and response to therapies in patients with GB.

In order to improve the understanding of the dynamics of the genomic alterations associated with tumor relapse, and to provide novel information on the aggressive behavior of GB tumors, primary GB tumors of patients with GB displaying short or long recurrence-free survival (RFS) outcome, were molecularly characterized and compared in the present study.

In particular, $19 \mathrm{~GB}$ samples were characterized according to the presence of mutations in the isocitrate dehydrogenase 1 (IDH1) gene, amplification of the epidermal growth factor receptor (EGFR) gene, presence of the EGFR variant III (EGFRvIII), and methylation of the promoter region of the $\mathrm{O}^{6}$-methylguanine-DNA methyltransferase (MGMT) gene. Furthermore, the expression of 84 genes known to be important for cell-cell and cell-matrix interactions, and that of 84 miRNAs known to switch their expression pattern during nervous system-associated carcinogenesis, were profiled. In addition, a copy number variation analysis of 23 genes, whose expression has been previously reported to be frequently altered in human glioma tumors (15), was also performed. 
Table II. Brain region localization, size (height $\mathrm{x}$ width $\mathrm{x}$ length) and area of the glioblastoma tumors in the short ( $\mathrm{n}=10)$ and long ( $\mathrm{n}=9)$ RFS cohorts.

\begin{tabular}{|c|c|c|c|c|}
\hline Surgical pathology number & RFS group & Brain region & Tumor size $(\mathrm{cm})$ & Tumor area $\left(\mathrm{cm}^{2}\right)$ \\
\hline 298/09 & Short & Parietal & $4.0 \times 3.0 \times 2.0$ & 52 \\
\hline $476 / 06$ & Long & Righ temporal & $5.5 \times 5.0 \times 2.0$ & 97 \\
\hline $2102 / 08$ & Long & $\mathrm{N} / \mathrm{A}$ & $2.0 \times 1.5 \times 1.0$ & 13 \\
\hline $3605 / 06$ & Short & Left frontal & $5.8 \times 4.5 \times 3.0$ & 114 \\
\hline $4096 / 10$ & Short & Frontal & $4.5 \times 4.0 \times 2.0$ & 70 \\
\hline $4239 / 12$ & Short & Right temporal & $4.0 \times 2.5 \times 1.0$ & 33 \\
\hline $4318 / 07$ & Long & Frontal & $2.5 \times 2.5 \times 2.0$ & 33 \\
\hline $4382 / 05$ & Short & Right insula & $1.4 \times 1.0 \times 0.8$ & 7 \\
\hline $4534 / 11$ & Long & $\mathrm{N} / \mathrm{A}$ & $3.0 \times 2.0 \times 0.5$ & 17 \\
\hline $4561 / 12$ & Long & $\mathrm{N} / \mathrm{A}$ & $5.0 \times 2.0 \times 0.3$ & 24 \\
\hline $4619 / 07$ & Long & Frontal & $1.2 \times 0.6 \times 0.5$ & 3 \\
\hline $5165 / 11$ & Long & $\mathrm{N} / \mathrm{A}$ & $2.0 \times 1.5 \times 1.0$ & 13 \\
\hline $6043 / 08$ & Long & $\mathrm{N} / \mathrm{A}$ & $5.0 \times 5.0 \times 2.0$ & 90 \\
\hline $7031 / 07$ & Long & Basal temporal lobe & $4.5 \times 3.5 \times 1.0$ & 48 \\
\hline $7624 / 05$ & Short & Right temporoparietal & $4.0 \times 3.6 \times 1.7$ & 55 \\
\hline $7901 / 09$ & Short & Left pre-rolandic (frontal) & $4.0 \times 2.0 \times 1.0$ & 28 \\
\hline 9298/07 & Short & Right frontal & $6.5 \times 5.0 \times 3.5$ & 146 \\
\hline $9303 / 09$ & Short & $\mathrm{N} / \mathrm{A}$ & $2.5 \times 2.0 \times 0.5+3.0 \times 3.0 \times 0.5$ & 27 \\
\hline $10504 / 10$ & Short & $\mathrm{N} / \mathrm{A}$ & $4.0 \times 3.0 \times 1.5$ & 45 \\
\hline
\end{tabular}

RFS, recurrence-free survival; N/A, not available.

\section{Materials and methods}

Patients and tumors. The present study included 19 newly diagnosed cases of GB, who presented to the Department of Translational Research and of New Surgical and Medical Technologies of the University Hospital of Pisa (Pisa, Italy). The patients, who were initially diagnosed by resection with GB grade IV (according to the World Health Organization classification criteria) (16), did not present any previous record of brain neoplasm. The GB tumors isolated from the patients were stored as formalin-fixed, paraffin-embedded (FFPE) specimens.

The GB cases included in the present study were selected according to their differences in progression-free survival. Of the 19 included GB cases, 10 cases, were classified as 'short RFS' and first displayed recurrence earlier than 6 months, and 9 cases, were classified as 'long RFS' and first displayed recurrence later than 14 months.

The median age of the patients was 61 years, and the gender distribution was 9/19 (47\%) males and 10/19 (53\%) females. The short RFS GB patients presented a median age of 63 years; gender distribution of $4 / 10(40 \%)$ males and $6 / 10(60 \%)$ females; and a mean RFS of 4.5 months, ranging from 2 to 6 months. The long RFS GB patients presented a median age of 58 years; gender distribution of 4/9 (44\%) males and 5/9 (56\%) females; and a mean RFS of 27 months, ranging between 14 and 71 months (Table I). The information regarding the size of the tumors and their localization within a certain brain region in relation to the duration of RFS is presented in Table II.
Ethical board. The present study was approved by the Internal Review Board of the University Hospital of Pisa. All patients provided their consent for participation in the study.

DNA and RNA extraction. DNA and RNA were extracted from $210-\mu \mathrm{m}$ sections of each FFPE specimen, using NucleoSpin Tissue (Machery-Nagel, Düren, Germany) and RNeasy FFPE Kit (Qiagen GmbH, Hilden, Germany) to extract the genomic DNA and total RNA from the tissues, respectively. The extracted DNA and RNA were quantified using Qubit 2.0 Fluorometer (Life Technologies, Grand Island, USA), according to the manufacturer's instructions. The DNA and RNA yields ranged from 50 to $500 \mathrm{ng} / \mu \mathrm{l}$.

Molecular markers. The samples were molecularly characterized by investigating the most studied molecular markers alterations in GB tumors, including mutations in the IDH1 gene; amplification of the EGFR gene; presence of EGFRvIII, the active mutant variant form of the EGFR gene; and methylation of the MGMT promoter:

IDH1 mutation. For the detection of IDH1 mutations, Primer3 software (http://primer3.ut.ee/) was used to design the following primers, in order to amplify the exon 4 of the IDH1 gene: Forward, 5'-AGCTCTATATGCCATCACTGC-3'; and reverse, 5'-TTCATACCTTGCTTAATGGGTGT-3'). Polymerase chain reaction (PCR) amplification was performed with AmpliTaq Gold DNA Polymerase with Buffer II and $\mathrm{MgCl}_{2}$ (Life Technologies), according to the manufacturer's protocol. The PCR products were loaded onto a $2 \%$ agarose gel, subjected to DNA gel electrophoresis, stained with ethidium 
bromide and verified by sequencing. For this purpose, the PCR bands were excised from the gel and purified using QIAquick Gel Extraction Kit (Qiagen $\mathrm{GmbH}$ ), prior to being subjected to direct sequencing in both directions with the aforementioned primers, using the BigDye Terminator v3.1 Cycle Sequencing Kit (Applied Biosystems Life Technologies, Foster City, USA). The sequencing reaction was conducted on an ABI PRISM 3130xl Genetic Analyzer (Applied Biosystems Life Technologies), according to the manufacturer's protocol.

Copy number variation. Analyses of the number of copies of the EGFR gene and the presence of the EGFRvIII variant were performed with SALSA MLPA P105 Glioma-2 probemix (MRC-Holland, Amsterdam, The Netherlands), according to the manufacturer's protocol. Fragment analysis was performed on an ABI PRISM 3130xl Genetic Analyzer (Applied Biosystems Life Technologies).

MGMT promoter methylation. Analysis of the methylation status of the MGMT promoter was performed with the MGMT plus kit (Diatech Pharmacogenetics, Jesi, Italy), according to the manufacturer's instructions. Following bisulfite conversion, the DNA was amplified with bisulfite-specific primers, using TaKaRa Ex Taq Hot Start DNA Polymerase (Clontech Laboratories, Inc., Mountainview, USA), according to the manufacturer's protocol, and analyzed on a PyroMark Q96 ID (Qiagen $\mathrm{GmbH}$ ), using Pyro Q-CpG software (Qiagen GmbH).

\section{PCR commercial arrays}

ECM and adhesion molecules. $100 \mathrm{ng}$ of isolated RNA was reverse transcribed to cDNA, and pre-amplified using the RT2 PreAMP cDNA Synthesis Kit (Qiagen $\mathrm{GmbH}$ ). The cDNA was aliquoted with Mastermix (Qiagen $\mathrm{GmbH}$ ) and added to the wells of an ECM microarray plate (catalogue no. PAHS-0013; SABiosciences, Frederick, USA), according to the manufacturer's instructions.

Brain cancer miRNA. $250 \mathrm{ng}$ of isolated RNA was reverse transcribed to cDNA using miScript II RT Kit (Qiagen $\mathrm{GmbH}$ ). The cDNA generated was subsequently used as a template for qPCR, using the miScript SYBR Green PCR Kit (SABiosciences, Qiagen $\mathrm{GmbH}$ ) and the Human Brain Cancer miRNA PCR Array (catalogue no. MIHS-108Z; SABiosciences), according to the manufacturer's instructions.

Glioma copy number. $800 \mathrm{ng}$ of extracted DNA was aliquoted with qBiomarker SYBR Mastermix (Qiagen $\mathrm{GmbH}$, Hilden, Germany) and added to the wells of a Human Glioma Copy Number PCR Array Plate (catalogue no. VAHS-0053Z, SABiosciences), according to the manufacturer's instructions.

All the above PCR array analyses were performed calculating the $\triangle \mathrm{CT}$ value for each gene profiled in the plate, using the formula $\Delta \mathrm{CT}=\mathrm{CT}$ (gene of interest) - average CT (reference gene). The fold difference value, used for statistical analysis, was equated to $2^{-\Delta \Delta \mathrm{CT}}$

Statistical analyses. The $\chi^{2}$ test was used to establish whether there was any association between the 2 categorical variables, short/long RFS group, and any alterations in molecular markers, including mutations in the IDH1 gene, EGFR amplification, presence of the mutant form EGFRvIII and methylation of the MGMT promoter. Differences in the gene expression levels between the 2 groups were analyzed by one-way analysis of variance. Discriminant analysis was conducted to
Table III. Differences in the expression levels of the 5 selected genes among the patients with glioblastoma, belonging to the short $(n=10)$ or long $(n=9)$ RFS groups.

\begin{tabular}{llll}
\hline & \multicolumn{2}{c}{ RFS (mean) } & \\
\cline { 2 - 3 } Gene & Short & Long & P-value \\
\hline miRNA-150-5p & 0.075 & 0.192 & $0.040^{\mathrm{a}}$ \\
miRNA-328-3p & 0.042 & 0.192 & 0.060 \\
SELE & 0.006 & 0.000 & 0.094 \\
SELL & 0.027 & 0.010 & 0.076 \\
ADAMTS13 & 0.013 & 0.002 & 0.059 \\
\hline
\end{tabular}

RFS, recurrence-free survival; mean, mathematical mean of $2^{-\Delta C T}$ gene expression levels within the group; ${ }^{a} \mathrm{P}<0.05$. miRNA, microRNA; SELE, selectin E; SELL, selectin L; ADAMTS13, a disintegrin and metalloproteinase with thrombospondin type 1 motif, member 13 .

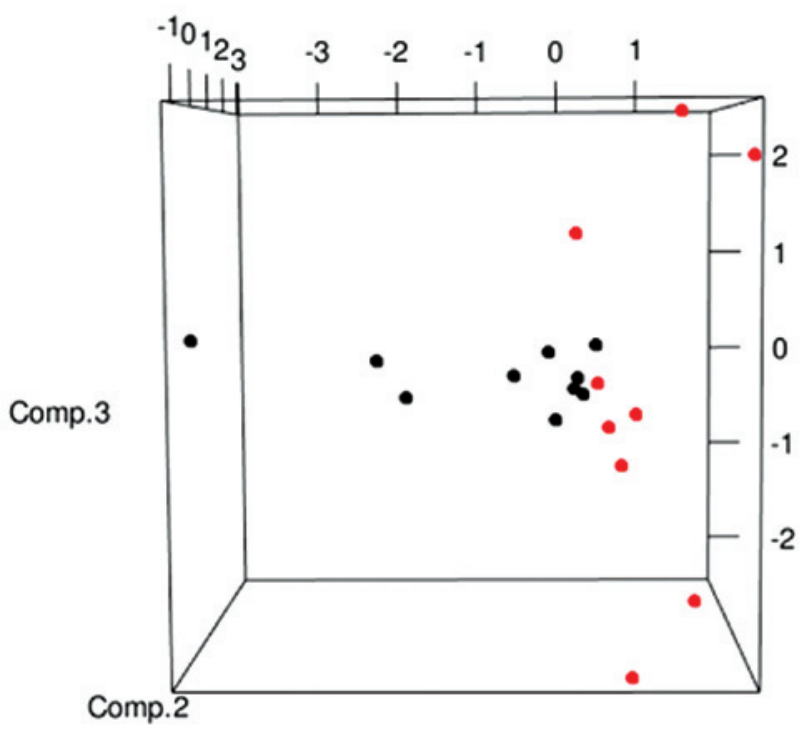

Comp. 1

Figure 1. Scatter-plot of principal component analysis. Visualization in a tridimensional space of the discriminative power of the expression levels of the 5 selected genes (microRNA-150-5p and -328-3p, selectin $\mathrm{E}$ and $\mathrm{L}$, and a disintegrin and metalloproteinase with thrombospondin type 1 motif, member 13), considered as variables, in correctly classifying the 19 GB samples included in the present study into their corresponding short or long RFS group. The black and red dots represent the 10 short and 9 long RFS GB samples, respectively. GB, glioblastoma; RFS, recurrence-free survival; Comp., component.

predict the probability of a particular sample to belong to the short or the long RFS group, based on the expression levels of the profiled genes exhibited by the sample.

Principal component analysis (PCA) was used to convert a set of observations of possibly correlated variables (gene expression levels) into a set of values of linearly uncorrelated variables termed PCs. PCs are guaranteed to be independent if the data set is jointly normally distributed (17). A tridimensional PCA scatter-plot was generated with R software 
Table IV. Classification of observations based on the derived discriminant functions.

A, Classification

\begin{tabular}{lclr} 
& & \multicolumn{2}{c}{ Predicted RFS, n (\%) } \\
\cline { 3 - 4 } Actual RFS & Actual RFS, total n & Short & Long \\
\hline Short & 10 & $9(90)$ & $1(10)$ \\
Long & 9 & $0(0)$ & $9(100)$ \\
\hline
\end{tabular}

B, Probabilities

\begin{tabular}{lccccc}
\hline Surgical pathology number & Actual RFS group & Predicted RFS group & Prob. & Second predicted RFS group & Prob. \\
\hline $298 / 09$ & Short & Short & 0.9989 & Long & 0.0011 \\
$476 / 06$ & Long & Long & 0.9912 & Short & 0.0088 \\
$2102 / 08$ & Long & Long & 0.9872 & Short & 0.0128 \\
$3605 / 06$ & Short & Short & 0.9992 & Long & 0.0008 \\
$4096 / 10$ & Short & Short & 0.8672 & Long & 0.1328 \\
$4239 / 12$ & Short & Short & 0.6459 & Long & 0.3541 \\
$4318 / 07$ & Long & Long & 0.9998 & Short & 0.0002 \\
$4382 / 05$ & Short & Short & 0.9962 & Long & 0.0038 \\
$4534 / 11$ & Long & Long & 0.5913 & Short & 0.4087 \\
$4561 / 12$ & Long & Long & 0.8025 & Short & 0.1975 \\
$4619 / 07$ & Long & Long & 0.6960 & Short & 0.3040 \\
$5165 / 11$ & Long & Long & 0.9139 & Short & 0.0861 \\
$6043 / 08$ & Long & Long & 0.9152 & Short & 0.0848 \\
$7031 / 07$ & Long & Long & 0.9983 & Short & 0.0017 \\
$7624 / 05$ & Short & Short & 0.9909 & Long & 0.0091 \\
$7901 / 09$ & Short & Short & 0.9753 & Long & 0.0247 \\
$9298 / 07$ & Short & Short & 0.8214 & Long & 0.1786 \\
$9303 / 09$ & Short & Long & 0.5591 & Short & 0.4409 \\
$10504 / 10$ & Short & Short & 0.9618 & Long & 0.0382 \\
\hline
\end{tabular}

Using 19 GB cases, a 5-genes combination approach was developed, in order to discriminate among the 2 types of RFS, short and long (considered as the classification variable), displayed by the patients. The 5 predictor variables entered were the gene expression levels of microRNA-150-5p and -328-3p, selectin E and L, and a disintegrin and metalloproteinase with thrombospondin type 1 motif, member 13. Of the 19 observations used, 18 (94.7\%) were correctly classified ( $\mathrm{P}=0.0079 ;{ }^{\text {a }}$ refers to the observations that were incorrectly classified). The actual, predicted and second predicted (non-predicted) statistical probability calculated for each GB sample of belonging to the short or long RFS group, is also indicated. RFS, recurrence-free survival; Prob., probability; GB, glioblastoma.

(R Foundation for Statistical Computing, Vienna, Austria; https://www.r-project.org/).

Leave-one-out cross-validation (LOOCV) was used as resampling method to estimate the prediction error of the model. Its indicator, the error rate, defined as 1 - accuracy rate, states the probability of misclassification of a classifier. In LOOCV, 1 instance is eliminated from the dataset, and the classifier is created on the remaining instances, in order to assess the correctness of the prediction of the eliminated instance. This process is then repeated for all the instances.

Logistic regression analysis was performed using Stata SE12.0 software (StataCorp LP, College Station, USA), considering short/long RFS group as the dependent variable, and the area of the tumor and the patient's age and gender as the independent variables.

\section{Results}

Demographic characteristics of the patients and molecular characterization of the tumors. Patients with GB were classified in 2 groups, based on their duration of RFS: Patients were defined as short RFS, if RFS $=<6$ months, and long RFS, if RFS $=>14$ months.

The comparison between the patient's characteristics, including age, gender and localization of the tumor, and the 2 lengths of RFS, did not identify any significant associations (data not shown). The calculated average tumor area was smaller in the long RFS group compared with the short RFS group (38 vs. $58 \mathrm{~cm}^{2}$, respectively; Table II), but this difference was not observed to be statistically significant (data not shown). 
Furthermore, using logistic regression analysis, the role of potential interference factors, including the patient's age, size of the tumor and extent of surgery, were excluded from the short/long RFS classification (data not shown).

Molecular markers. Codon 132 in the IDH1 gene was confirmed to be wild-type in all the $19 \mathrm{~GB}$ samples analyzed, while 9/19 (44\%) GB samples presented with EGFR amplification, and 3/9 (33\%) samples carried the EGFRvIII variant (Table I). Among the short RFS samples, 3/10 (30\%) cases presented with EGFR amplification, and 1/3 (33\%) carried the EGFRvIII variant; whereas in the long RSF samples, 6/9 (67\%) cases presented with EGFR amplification, and 2/6 (33\%) carried the EGFRvIII variant (Table I). Overall, 8/19 (42\%) GB samples exhibited MGMT promoter methylation, 5 of which belonged to the 10 short RFS group (50\%), whereas only $3(33 \%)$ of those samples with MGMT promoter methylation belonged to the 9 long RSF group (Table I).

Molecular markers and RFS association analysis. The comparison between the frequencies of alterations in the molecular marker that were identified in the patients with GB, and the differences in RFS displayed by these patients, did not reveal any significant differences. Furthermore, no significant correlations were observed among all the molecular alterations analyzed.

PCR array data and RFS association analysis. The differences between the short and long RFS groups, regarding the expression levels and copy number of 191 genes, were analyzed using 3 PCR arrays. The results revealed significant differences in the expression levels of miRNA-150-5p between the 2 groups $(\mathrm{P}=0.040$; Table III).

5-genes combination. In order to perform a discriminant analysis and predict the probability of a GB case to belong to the short or long RFS group, based on their gene expression levels, 5 genes with the lowest $\mathrm{P}$-value $(\mathrm{P}<0.095)$, including miRNA-150-5p and -328-3p, selectin E (SELE) and L (SELL), and a disintegrin and metalloproteinase with a thrombospondin type 1 motif, member 13 (ADAMTS13), were selected for the analysis (Table III).

The combination of the expression levels of the above 5 genes was able to correctly classify the GB samples into their corresponding short or long RFS group, with a precision of 90 and $100 \%$, respectively (Table IV). Overall, $94.74 \%$ (18/19) GB cases were correctly classified with this method $(\mathrm{P}=0.008$; Table IV). The probability of each sample to belong to one or the other group is also indicated in Table IV. Among the 18 samples correctly classified, $12(66 \%)$ were assigned a probability $>90 \%$, including 7 cases $(34 \%)$ whose probability was $>99 \%$ (Table IV). Using the expression levels of the aforementioned 5 selected genes (miRNA150-5p, miRNA328-3p, SELE, SELL and ADAMTS13) as data variables, a PCA was performed, which clearly indicated a well-defined separation of the 2 RFS populations in a tridimensional space (Fig. 1).

Cross-validation of the 5-genes combination. The prediction error of the 5-genes combination approach was estimated via cross-validation, using a resampling method. In this type
Table V. Overall cross-validation error rate.

A, Value prediction

\begin{tabular}{lcc}
\hline RFS & Recall & 1-precision \\
\hline Short & 0.9000 & 0.1818 \\
Long & 0.7778 & 0.1250 \\
\hline
\end{tabular}

B, Confusion matrix

\begin{tabular}{lccr}
\hline RFS & Short & Long & Total \\
\hline Short & 9 & 1 & 10 \\
Long & 2 & 7 & 9 \\
Total & 11 & 8 & 19
\end{tabular}

Overall cross-validation of the 5-genes combination approach using the leave-one-out method. Recall is the percentage of correctly predicted instances, whereas 1-precision is the percentage of actual errors among all the encounters classified as errors. The error rate is the percentage of datasets that are incorrectly classified by the method, which corresponds to the probability of misclassification of a classifier. The error rate of this 5-genes combination approach was determined to be 0.1579 for the predicted values. RFS, recurrence-free survival.

of methodology, the prediction error is calculated for each measured tree from a presumed distribution of errors, and a dataset is subsequently generated as the sum of a prediction and a random prediction error.

The percentage of cases correctly classified following this method was $90 \%$ (9/10) in the short RFS samples, and $78 \%$ (7/9) in the long RFS samples, with a total error rate of 0.1579 (Table V).

\section{Discussion}

In regards to GB, it is of importance to identify key molecular markers, and to perform simple and prompt molecular diagnostic tests that are able to predict the survival time and therapeutic response of patients. In the present study, a wide molecular characterization, including analysis of miRNA, RNA and DNA, was performed on 19 GB cases with different RFS outcome, in an attempt to better understand the dynamics of the genomic alterations associated with relapse in patients with GB. A long-term recurrence GB group, with an RFS ranging from 14 to 71 months (mean RFS $=27$ months), was included in the study. This group represents rare cases of GB, compared with the GB RFS reported in the literature (3).

Based on the clinical history of the patients and the molecular features of their tumors, all the cases were classified as primary GBs. The patients did not present any previous records of brain neoplasm, and their median age at the time of diagnosis was 60 years, which is in agreement with previous studies that reported the mean age of patients clinically diagnosed with primary GB to be 62 years, and 45 years in the case of patients clinically diagnosed with secondary GB (18). In addition, the patients of the present 
study exhibited a high frequency of overexpression of EGFR (44\%) and absence of IDH1 mutations, which are typical features of primary GBs (18).

The expression levels of 84 miRNAs whose expression pattern is altered during nervous system-associated carcinogenesis, and 84 genes whose products are involved in cell-cell and cell-matrix interaction processes, were evaluated by qPCR array. Additionally, a copy number PCR array analysis was performed on 23 genes whose expression pattern has been reported to be frequently altered in human glioma tumors (15). The results revealed significant differences in the expression levels of miRNA150-5p between the short and long RFS groups.

The 5 genes with the highest differences in gene expression levels that were identified in the study, including miRNA150-5p, miRNA328-3p, SELE, SELL and ADAMTS13, were selected for subsequent analysis, despite their P-values were not significant $(\mathrm{P}<0.095)$. The resulting 5 -genes combination approach was able to correctly classify $94.74 \%$ of the 19 GB samples into their corresponding short or long RFS groups. To the best of our knowledge, the expression pattern of the above 5 genes has never been associated with RFS in GB thus far.

Next, an LOOCV was performed to evaluate the performance of this 5-genes combination approach, and a low error rate $(0.1579)$ was obtained for this method, indicating that $84 \%$ of the 19 GB cases were correctly classified following this approach.

Of the aforementioned genes, SELE, SELL and ADAMTS13 were observed to be overexpressed in the short RFS GB samples, which is the group with the worst prognosis. ADAMTS13 has been previously associated with the degradation and remodeling of the ECM, while SELE and SELL are cell adhesion molecules (CAMs) that mediate cell attachment, migration and signaling to and from the ECM (15). Alterations of CAMs have been reported to be common in cancer, where the disruption of cell-cell or cell-ECM adhesion significantly contributes to uncontrolled cell proliferation and progressive distortion of the architecture of normal tissue (19). Furthermore, alterations in CAMs have been involved in tumor dissemination, since the loss of cell-cell adhesion enables the malignant cells to detach and subsequently migrate from the primary mass (19).

By contrast, miRNA-150-5p and 328-3p were observed to be overexpressed in the long RFS GB group, which is the group with the best prognosis. Previous studies have demonstrated that the increased expression of miR-150 inhibited breast cancer cell migration and invasion in vitro, and decreased cell proliferation and induced apoptosis in natural killer and T-cells $(20,21)$. A previous study revealed that patients with GB whose expression levels of miR-328 were lower than the median value, exhibited poor survival, since miR-328 is associated with the cell cycle progression (22). Therefore, the ectopic expression of miR-328 in GB cells may suppress cell proliferation (22).

In the present study, the GB samples were divided and compared, according to their short or long RFS time, despite not being 2 distinct clinical or pathological entities. All the patients with GB displayed the same histological and pathological features, including high malignancy, invasive behavior and poor prognosis, regardless of their short or long RFS. Therefore, it is not easy to differentiate between short and long RFS in patients with GB. However, it is of importance to understand the mechanisms leading to aggressive GB tumors with high spreading capacities, which are not susceptible to standard treatments, including surgery, radiotherapy and chemotherapy, whereas other GB tumors are more manageable, and enable patients to live $>1$ year. Although tumor invasion and metastasis are known to be the main causes of mortality in patients with solid cancer, including GB, the characterization of the molecular and cellular mechanisms behind these processes is limited.

In conclusion, the present study has demonstrated that alterations in the expression pattern of certain miRNAs, mRNAs and genomic DNA constitute a particular molecular hallmark, which may increase or reduce the aggressive behavior of GB tumors, thus influencing the survival time, response to therapy and tendency to experience a tumor relapse of patients with GB. In particular, the 5-genes combination approach performed in the present study was able to correctly predict the short or long RFS outcome in the majority of patients with GB. The high sensitivity and precision of this approach, as confirmed by LOOCV, provide a strong foundation for further validation of the association between alterations in the expression of the aforementioned 5 genes and the RFS of patients with GB in a larger population.

\section{References}

1. Stupp R, Mason WP, van den Bent MJ, Weller M, Fisher B, Taphoorn MJ, Belanger $\mathrm{K}$, Brandes AA, Marosi C, Bogdahn U, et al; European Organisation for Research and Treatment of Cancer Brain Tumor and Radiotherapy Groups; and National Cancer Institute of Canada Clinical Trials Group: Radiotherapy plus concomitant and adjuvant temozolomide for glioblastoma. N Engl J Med 352: 987-996, 2005.

2. Grossman SA, Ye X, Piantadosi S, Desideri S, Nabors LB, Rosenfeld M and Fisher J; NABTT CNS Consortium: Survival of patients with newly diagnosed glioblastoma treated with radiation and temozolomide on research studies in the United States. Clin Cancer Res 16: 2443-2449, 2010.

3. Hou LC, Veeravagu A, Hsu AR and Tse VC: Recurrent glioblastoma multiforme: A review of natural history and management options. Neurosurg Focus 20: E5, 2006.

4. Wong ET, Hess KR, Gleason MJ, Jaeckle KA, Kyritsis AP, Prados MD, Levin VA and Yung WK: Outcomes and prognostic factors in recurrent glioma patients enrolled onto phase II clinical trials. J Clin Oncol 17: 2572-2578, 1999.

5. Lamborn KR, Yung WK, Chang SM, Wen PY, Cloughesy TF, DeAngelis LM, Robins HI, Lieberman FS, Fine HA, Fink KL, et al; North American Brain Tumor Consortium: Progression-free survival: An important end point in evaluating therapy for recurrent high-grade gliomas. Neuro Oncol 10: $162-170,2008$.

6. Zhang W, Zhang J, Yan W, You G, Bao Z, Li S, Kang C, Jiang C, You Y, Zhang Y, et al: Whole-genome microRNA expression profiling identifies a 5-microRNA signature as a prognostic biomarker in Chinese patients with primary glioblastoma multiforme. Cancer 119: 814-824, 2013.

7. Knott JC, Mahesparan R, Garcia-Cabrera I, Bølge Tysnes B, Edvardsen K, Ness GO, Mørk S, Lund-Johansen M and Bjerkvig R: Stimulation of extracellular matrix components in the normal brain by invading glioma cells. Int J Cancer 75: 864-872, 1998.

8. Freije WA, Castro-Vargas FE, Fang Z, Horvath S, Cloughesy T, Liau LM, Mischel PS and Nelson SF: Gene expression profiling of gliomas strongly predicts survival. Cancer Res 64: 6503-6510, 2004.

9. Phillips HS, Kharbanda S, Chen R, Forrest WF, Soriano RH, Wu TD, Misra A, Nigro JM, Colman H, Soroceanu L, et al: Molecular subclasses of high-grade glioma predict prognosis, delineate a pattern of disease progression, and resemble stages in neurogenesis. Cancer Cell 9: 157-173, 2006.

10. Berens ME and Giese A: '...those left behind.' Biology and oncology of invasive glioma cells. Neoplasia 1: 208-219, 1999. 
11. Besse A, Sana J, Fadrus P and Slaby O: MicroRNAs involved in chemo- and radioresistance of high-grade gliomas. Tumour Biol 34: 1969-1978, 2013.

12. Ambros V: MicroRNAs: Tiny regulators with great potential. Cell 107: 823-826, 2001.

13. Yin AA, Zhang LH, Cheng JX, Dong Y, Liu BL, Han N and Zhang X: The predictive but not prognostic value of MGMT promoter methylation status in elderly glioblastoma patients: A meta-analysis. PLoS One 9: e85102, 2014.

14. Colen RR, Vangel M, Wang J, Gutman DA, Hwang SN, Wintermark M,Jain R, Jilwan-Nicolas M, Chen JY, Raghavan P, et al; TCGA Glioma Phenotype Research Group: Imaging genomic mapping of an invasive MRI phenotype predicts patient outcome and metabolic dysfunction: A TCGA glioma phenotype research group project. BMC Med Genomics 7: 30, 2014.

15. Qiagen: Glioma Copy Number PCR Array. http://www.sabiosciences.com/copynumber_product/HTML/VAHS-0053Z.html.

16. Louis DN, Ohgaki H, Wiestler OD, Cavenee WB, Burger PC, Jouvet A, Scheithauer BW and Kleihues P: The 2007 WHO classification of tumours of the central nervous system. Acta Neuropatho 114: 97-109, 2007.
17. Jolliffe IT: Springer Series in Statistics: Principal Component Analysis. 2nd edition. Springer, NY, p28, 2002. ISBN 978-0-387-95442-4

18. Ohgaki $\mathrm{H}$ and Kleihues $\mathrm{P}$ : The definition of primary and secondary glioblastoma. Clin Cancer Res 19: 764-772, 2013.

19. Moh MC and Shen S: The roles of cell adhesion molecules in tumor suppression and cell migration: A new paradox. Cell Adhes Migr 3: 334-336, 2009.

20. Huang S, Chen Y, Wu W, Ouyang N, Chen J, Li H, Liu X, Su F, Lin $\mathrm{L}$ and Yao Y: miR-150 promotes human breast cancer growth and malignant behavior by targeting the pro-apoptotic purinergic P2X7 receptor. PLoS One 8: e80707, 2013.

21. Caramuta S,Lee L, OzataDM,AkçakayaP,Georgii-Hemming P Xie H, Amini RM, Lawrie CH, Enblad G, Larsson C, et al: Role of microRNAs and microRNA machinery in the pathogenesis of diffuse large B-cell lymphoma. Blood Cancer J 3: e152, 2013

22. Wu Z, Sun L, Wang H, Yao J, Jiang C, Xu W and Yang Z: MiR-328 expression is decreased in high-grade gliomas and is associated with worse survival in primary glioblastoma. PLoS One 7: e47270, 2012. 